This is the final peer-reviewed accepted manuscript of:

Zuccarini, M., Guarini, A., Savini, S., Iverson, J. M., Aureli, T., Alessandroni, R., Faldella, G., \& Sansavini, A. (2017). Object exploration in extremely preterm infants between 6 and 9 months and relation to cognitive and language development at 24 months. Research in Developmental Disabilities, 68, 140-152.

The final published version is available online at:

https://doi.org/10.1016/j.ridd.2017.06.002

Rights / License:

The terms and conditions for the reuse of this version of the manuscript are specified in the publishing policy. For all terms of use and more information see the publisher's website.

This item was downloaded from IRIS Università di Bologna (https://cris.unibo.it/)

When citing, please refer to the published version. 


\title{
Object exploration in extremely preterm infants between 6 and 9 months and relation to cognitive and language development at 24 months
}

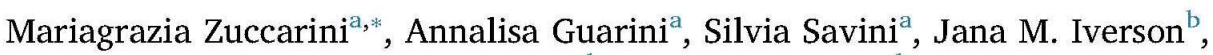 \\ Tiziana Aureli ${ }^{\mathrm{c}}$, Rosina Alessandroni ${ }^{\mathrm{d}}$, Giacomo Faldella ${ }^{\mathrm{d}}$, Alessandra Sansavini ${ }^{\mathrm{a}}$ \\ a Department of Psychology, University of Bologna, Via Berti Pichat, 5-40127 Bologna, Italy \\ ${ }^{\mathrm{b}}$ Department of Psychology, University of Pittsburgh, USA \\ ' Department of Neuroscience, Imaging and Clinical Sciences, University of Chieti-Pescara, Italy \\ a Neonatology and Neonatal Intensive Care Unit - S. Orsola-Malpighi Hospital, Department of Medical and Surgical Sciences, University of Bologna \\ Italy
}

\section{A R T I C L E I N F O}

\section{Keywords:}

Preterm infants

Object exploration

First year of life

Fine motor abilities

Precursors of cognitive and language

development

\begin{abstract}
A B S T R A C T
Although early object exploration is considered a key ability for subsequent achievements, very few studies have analyzed its development in extremely low gestational age infants (ELGAGA $<28$ weeks), whose early motor skills are delayed. Moreover, no studies have examined its developmental relationship with cognitive and language skills. The present study examined developmental change in Motor Object Exploration (MOE) and different types of MOE (Holding, Oral, Manual and Manual Rhythmic Exploration) in 20 ELGA and 20 full term (FT) infants observed during mother-infant play interaction at 6 and 9 months. It also explored whether specific types of MOE were longitudinally related to 24-month language and cognitive abilities (GMDS-R scores). ELGA infants increased MOE duration from 6 to 9 months, eliminating the initial difference with FT infants. In addition, ELGA infants showed a different pattern of Oral Exploration, that did not increase at 6 months and decrease at 9 months. Oral and Manual Exploration durations at 6 months were longitudinally related to 24-month GMDS-R language and cognitive performance scores respectively. We discuss the relevance of assessing early exploratory abilities in ELGA infants in order to implement customized intervention programs for supporting the development of these skills.
\end{abstract}

\section{What this paper adds?}

The motor domain is particularly vulnerable in extremely low gestational age infants (ELGA $<28$ weeks) whose motor skills are delayed from the outset, even in absence of brain damage. Among early fine-motor abilities, very few studies have analyzed the development of object exploration in this population, although this is considered a key ability for subsequent achievements. Moreover, no studies have examined its developmental relationship with cognitive and language. This paper adds a detailed description of the longitudinal change of the exploratory abilities in ELGA infants from 6 to 9 months and explore whether specific

\footnotetext{
* Corresponding author.

E-mail addresses: mariagrazia.zuccarini@unibo.it (M. Zuccarini), annalisa.guarini@unibo.it (A. Guarini), silvia.savini3@unibo.it (S. Savini), jiverson@pitt.edu (J.M. Iverson), t.aureli@unich.it (T. Aureli), rosina.alessandroni@aosp.bo.it (R. Alessandroni), giacomo.faldella@unibo.it (G. Faldella), alessandra.sansavini@unibo.it (A. Sansavini).
} 
exploratory behaviors at 6 months are related to 24-month language and cognitive abilities. The findings reveal that ELGA infants show different developmental patterns in object exploration compared to FT infants. In particular, they increase Motor Object Exploration duration from 6 to 9 months but, differently from FT infants, they do not show an increase in Oral Exploration at 6 months and a decrease at 9 months. In addition, our results show that 6-month duration of Oral and Manual Exploration contribute to predict the 24-month Hearing \& Language and cognitive Performance scores, respectively, suggesting that early motor abilities are closely related to cognitive and language development. Considering the potential cascading effects that subtle difficulties in object exploration may have on language and cognitive development, our findings point out the relevance of assessing early exploratory abilities in ELGA infants in order to implement customized intervention programs for supporting the development of these skills.

\section{Introduction}

Although advances in neonatal intensive care have improved the survival rate of extremely low gestational age infants (ELGAborn $<28$ weeks of gestation) in the last two decades, the risk of cognitive, language and motor impairments in this population remains a concern, with an increasing likelihood of adverse developmental outcomes in multiple domains (Anderson \& Doyle, 2008; de Kievet, Piek, Aarnoudse-Moens, \& Oosterlaan, 2009; Marlow, Wolke, Bracewell, \& Samara, 2005; Sansavini, Savini, Guarini et al., 2011; Sansavini et al., 2014; Wolke \& Meyer, 1999).

In the motor domain, some studies have revealed that compared to full-term (FT) infants, ELGA infants show slower developmental trajectories and a higher number of difficulties, even in the absence of cerebral abnormalities. These differences are evident by the first year of life and persist until adulthood (de Kievet et al., 2005; Hille et al., 2008; Husby, Skranes, Olsen, Brubakk, \& Evensen, 2013; Sansavini et al., 2014; Sansavini, Savini, Guarini et al., 2011). Motor difficulties could be also detrimental for development in other domains, since, as described in several studies on typically developing (TD) infants, the achievement and improvement of motor skills at an early age may afford later advances in linguistic and cognitive functions. In particular, the infant capacity for action can offer a unique contribution in that direction. Iverson (2010) noted that early motor achievements, including object exploration, provide infants with the opportunity to practice skills that are critically shared with language, and allow infants to experiment changes in the relationships with objects and people that are relevant for communicative development in general and for language acquisition in particular. In support of this suggestion, research has found that early manipulative practice related significantly with larger vocabulary and psychomotor general scores at later age (Ruddy \& Bornstein, 1982); moreover, it has also found that the effects of motor abilities on development persist until childhood, as revealed by Oudgenoeg-Paz, Leseman, and Volman (2014) with respect to early spatial exploration as related to later spatial memory, and until adolescence, as showed by Bornstein, Hahn, Suwalsky (2013), with respect to the extent and efficiency of early exploration as related to later academic achievement.

Although these studies are correlational and do not permit causal conclusions, they underscore the existence of a significant relationship between exploratory skills and later development. However, very few studies have analyzed exploratory skills in the ELGA population, and the nature of this relationship remains largely unexplored. The aim of the current study is to address this gap in the literature by examining developmental change in object exploration behaviors in ELGA and FT infants between 6 and 9 months of age and by analyzing developmental relationships between specific exploratory behaviors observed at 6 months and language and cognitive abilities assessed at 24 months.

\subsection{Object exploration in TD and preterm infants}

From an early age, TD infants actively explore objects in their environment. Although exploratory behaviors are first observable at 2 months of age (Lobo, Kokkoni, de Campos, \& Galloway, 2014; Rochat, 1989), they consistently improve with the onset of reaching and the emergence of the sitting posture around 4-5 months of age (Berthental \& Von Hofsten, 1998; Lobo \& Galloway, 2013; Rochat \& Goubet, 1995). The achievement of these milestones promotes significant changes in object exploration, increasing the number, variety, and duration of the available behaviors (Rochat, 1989). Some studies have shown that 6 month-old infants exhibit different types of object exploration based on object affordances (Lobo et al., 2014), thereby obtaining different perceptual information based on object characteristics (Bushnell \& Boudreau, 1993; Palmer, 1989; Rochat, 1989; Ruff, 1984). In addition, the frequency of exploratory behaviors changes over time. In particular, Oral Exploration (e.g. mouthing) shows higher frequency and duration at 6 months, then decreases between 6 and 9 months (Belsky \& Most, 1981; Lobo et al., 2014; Ruff, 1984); Manual exploratory behaviors (e.g. transferring, turn rotating, and fingering) increase over time, reaching higher frequency and duration between 6 and 9 months (Lobo et al., 2014; Ruff, 1984); Manual Rhythmic behaviors such as banging and shaking increase before 6 months and do not change significantly between 6 and 9 months (Lobo et al., 2014).

As noted previously, the development of object exploration has been studied in TD infants, whereas less it is known about object exploration in infants at risk for delays in motor development, such as preterm infants. The studies that addressed this issue are few in number and have included preterm infants with neurological damage and across a wide range of gestational ages. Kopp (1976) found that preterm infants without neurological damage spent similar proportions of time manipulating objects as FT infants at 8 months, but types of exploration were different, with preterm infants spending less time mouthing than their FT counterparts. Unfortunately, the gestational ages and the use of corrected age in the preterm group were not specified; thus, the findings of this study cannot be generalized. Another study (Ruff, McCarton, Kurtzberg, \& Vaughan, 1984), which examined preterm infants with a gestational age $<34$ weeks, reported that preterm infants with neurological damage (high risk) showed less advanced object exploration than preterm infants without neurological damage (low risk) and FT infants at 9 months corrected age, with a shorter time spent in transferring, turn rotating, and fingering. No differences were found between low risk preterm infants and FT peers. In these studies, 
object exploration was assessed at around 9 months; however, data on TD infants suggest that exploratory behaviors are observable earlier in development with frequency and duration changing over time.

A more recent study (Lobo, Kokkoni, Cunha, \& Galloway, 2015), overcame these limitations and analyzed some specific exploratory behaviors, i.e., holding, mouthing, banging and their combinations, in very preterm infants (gestational age $<30$ weeks) with or without significant brain injury and in FT peers, longitudinally from birth to 24 months of corrected age. The results of this study confirmed that preterm infants explore objects for a shorter time and with a reduced variety of exploratory behaviors compared to FT infants throughout the first 2 years of life. These differences were more pronounced in the first six months of life and were more marked in those preterm infants with brain injury, who showed less banging, fingering and cyclical movements relative to the other groups. These findings highlighted the fact that cerebral damages in preterm infants contribute to delay in the development of object exploration.

Studies specifically examining the consequences of extreme preterm birth without cerebral damage on the development of object exploration are rare. The few existing studies reported that ELGA infants with cerebral integrity show lower scores in gross and fine motor skills and in cognitive performance compared to FT infants by 6 months of age (Sansavini, Savini, Guarini et al., 2011), and that differences in motor, cognitive, and language development persist throughout adulthood (de Kievet et al., 2009; Hille et al., 2008; Husby et al., 2013; Sansavini et al., 2014). Despite this evidence, only one recent study (Zuccarini et al., 2016) analyzed object exploration in ELGA infants without neurological damage. This study revealed that ELGA infants actively explored (i.e., mouthed and turn rotated) objects for a shorter time compared to FT infants at 6 months. However, because no studies to date have examined object exploration and specific types of exploratory behaviors longitudinally in ELGA infants, our understanding of how this skill develops in this population and whether some key differences in their exploratory behaviors persist over time is very limited.

\subsection{Relationship between object exploration and cognitive and language development in TD and preterm infants}

Object exploration is considered a foundational ability for later development across domains, as infants who explore longer and with a larger variety of exploratory behaviors create more opportunities for gathering information and acquiring knowledge on object characteristics (e.g., texture, shape, weight, and overall structure; Baumgartner \& Oakes, 2013; Gibson, 1988; Lederman \& Klatzky, 1993; Lobo et al., 2014; Ruff, 1984). Some studies have shown that object exploration promotes improvement in object individuation, i.e., the ability to represent the world in terms of distinct objects that persist in space and time (Needham, 2000; Wilcox et al., 2007), which constitutes a fundamental achievement for subsequent cognitive and language development (Oakes \& Madole, 2000). Ruddy and Bornstein (1982) found that babies who manipulated objects more frequently at 4 months had significantly larger vocabularies and obtained higher scores on mental and motor development assessed with the Bayley-I Scales at 12 months. Siegel (1981) showed that manipulation assessed at 6 months was related to language and cognitive performance at 2 years. More recent studies have provided evidence that the relationships between object exploration and subsequent competencies last also in the long term. Indeed, a study has showed that spatial exploration abilities in the first two years of age facilitated acquisition of spatial cognition at 4 and 6 years of age (Oudgenoeg-Paz et al., 2014). Another study found that exploration, in terms of its extent and efficiency, at 5 months of age was related to intellectual functioning at 4 and 10 years of age and to academic achievement at 10 and 14 years (Bornstein et al., 2013).

Beyond the overall frequency and duration of object exploration, there is also evidence that frequency and duration of specific active exploratory behaviors may trigger cognitive and language development. For instance, a study by Fagan and Iverson (2007) revealed that TD infants, both at 6 and 9 months, vocalized more frequently and produced more vocalizations with consonants during object mouthing.

In the preterm population, a study showed that Manual exploratory behaviors (i.e., fingering, transferring, and turn rotating) observed at 9 months were related to cognitive development at 24 months in high-risk preterm infants (Ruff et al., 1984). Along these lines, the only study conducted on ELGA infants revealed a significant concurrent relationship at 6 months between the duration of more advanced exploration behaviors, general psychomotor development, and eye-hand coordination assessed with the GMDS-R (Zuccarini et al., 2016). Although the studies cited above have shown that active object exploration is in some way related to cognitive and language skills, they did not draw a clear conclusion about the existence of a relationship between them.

\subsection{The present study}

The present study was designed to address two primary aims. The first was to describe object exploration longitudinally from 6 to 9 months in ELGA infants without cerebral abnormalities compared to FT infants. We observed spontaneous mother-infant play and analyzed how much time infants spent exploring objects via passive exploration (e.g., Holding objects) or active exploration (e.g., Oral, Manual, and Manual Rhythmic behaviors) which, as previously illustrated, fulfill different functions and show different developmental trajectories between 6 and 9 months in TD infants. Clinical assessments of cognitive, language, and motor skills at 6 and 9 months were also obtained using a standardized tool, the Griffiths Mental Developmental Scale (GMDS-R, Griffith, 1996) in order to compare psychomotor development between the two groups. Based on previous research (Sansavini, Savini, Guarini et al., 2011; Zuccarini et al., 2016) we hypothesized that ELGA infants would spend a lower percentage of time in active manual exploration and show different developmental change in this activity between 6 and 9 months compared to their FT peers. Less advanced psychomotor development was also expected in the ELGA sample.

The second aim was to investigate whether specific exploratory behaviors (i.e., Oral and Manual) at 6 months, which are related to language and cognitive achievements in TD infants, may explain an additional amount of variance in language and cognitive skills 
Table 1

Biological, socio-demographic and medical characteristics of the two groups.

\begin{tabular}{|c|c|c|}
\hline & ELGA $(\leq 28$ weeks $)(n=20)$ & FT $(>37$ weeks $)(n=20)$ \\
\hline $\mathrm{GA}$, mean (SD), range (weeks) & 25.9 (1.5) $23.2-28.5$ & 39.5 (1.5) $37-42$ \\
\hline $\mathrm{BW}$, mean (SD), range $(\mathrm{g})$ & 803 (191) 611-994 & 3340 (445) $2894-3784$ \\
\hline Male, $n(\%)$ & $9(45)$ & $9(45)$ \\
\hline First born, $n(\%)$ & $15(75)$ & $15(75)$ \\
\hline Later born, $n(\%)$ & $5(25)$ & $5(25)$ \\
\hline Mothers with a middle/low educational level, $n$ (\%) & $12(60)$ & $9(45)$ \\
\hline Mothers with a high educational level, $n(\%)$ & $8(40)$ & $11(55)$ \\
\hline Maternal age, mean (SD), range (years) & $36.2(4.8) 27-44$ & $34.4(3.0) 30-45$ \\
\hline Caesarean section, $n(\%)$ & $17(85)$ & $3(15)$ \\
\hline Multiple births, $n(\%)$ & $6(30)$ & $2(10)$ \\
\hline $\mathrm{BW}<1000 \mathrm{~g}, n(\%)$ & $16(80)$ & $0(0)$ \\
\hline $\mathrm{MV}, n(\%)$ & $11(55)$ & $0(0)$ \\
\hline $\mathrm{SGA}, n(\%)$ & $2(10)$ & $0(0)$ \\
\hline RDS, $n(\%)$ & $20(100)$ & $0(0)$ \\
\hline Apnoea, $n(\%)$ & $6(30)$ & $0(0)$ \\
\hline $\mathrm{BPD}, n(\%)$ & $12(60)$ & $0(0)$ \\
\hline IVH $\mathrm{I} / \mathrm{II}, \boldsymbol{n}(\%)$ & $1(5)$ & $0(0)$ \\
\hline HE $(\geq 14 d), n(\%)$ & $17(85)$ & $0(0)$ \\
\hline ROP I/II, $n(\%)$ & $13(65)$ & $0(0)$ \\
\hline Sepsis, $n(\%)$ & $6(30)$ & $0(0)$ \\
\hline Hyperbil.with phototer., $n$ (\%) & $16(80)$ & $0(0)$ \\
\hline
\end{tabular}

GA, gestational age; BW, birthweight; MV, mechanical ventilation.

Medical complications (infants could have one or more medical complication): SGA: infants with a birthweight < 10th percentile for gestational age; RDS: respiratory distress syndrome, acute illness coming on within 4-6 h of delivery, characterized clinically by respiratory rate $\geq 60 / \mathrm{min}$, dysponea and respiratory distress; Apnoea: significant apnoea was defined as more than four episodes of apnoea/hour or more than two episodes of apnoea/hour if ventilation with bag and mask was required, BPD: bronchopulmonary dysplasia, need of both supplemental oxygen for $\geq 28$ days and at 36 weeks of post-conceptional age; IVH I/II: intra-ventricular haemorrhage originating within the subependymal germinal matrix filling less than respectively $10 \%$ (I grade) and $50 \%$ (II grade) of the ventricular area on parasagittal view; HE: hyperecogenicity, a prolonged flare $\geq 14$ days without cystic lesions and/or ventricular dilatation and that resolved completely without any abnormality in its place; ROP I/II: retinopathy of prematurity, vasoproliferative retinopathy that resolved without a specific therapy before the presume date of birth; Sepsis: presence of a positive blood culture and/or clinical and laboratoristic signs; Hyperbil.with phototer:: hyperbilirubinemia needing phototherapy according to the criteria proposed by Gomella (2009).

measured with the GMDS-R scores at 24 months, above and beyond the amount of variance explained by neonatal status (ELGA vs. FT) and standardized language and cognitive scores obtained at 6 months. We focused on 6 months as the first point of assessment because ELGA infants show less advanced object exploration compared to FT infants by this age (Zuccarini et al., 2016) and on 24 months as the outcome point since cognitive difficulties become more evident at this age and assessment measures are more stable. Consistent with previous research, which found that Oral Exploration promoted the production of vocalizations at 6 and 9 months (Fagan \& Iverson, 2007), and Manual Exploration at 9 months was related to cognitive development at 24 months (Ruff et al., 1984), we hypothesized that the durations of Oral and Manual Exploration at 6 months would be longitudinally related to 24-month language and cognitive skills respectively, evaluated with the GMDS-R.

\section{Method}

\subsection{Participants}

Twenty ELGA infants and 20 FT monolingual Italian infants were enrolled in this longitudinal study. ELGA infants were born at the Neonatal Intensive Care Unit (NICU) of the University of Bologna, in the Emilia-Romagna region of Italy. They were recruited if, at birth, they had met three primary medical criteria: (a) gestational age (GA) $\leq 28$ weeks, determined by the date of the mother's last menstrual period and confirmed by first-trimester early ultrasonography; (b) no indication of major cerebral damage as detected by ultrasound scan (US) and confirmed by magnetic resonance imaging at 40 weeks of GA when its employment was indicated by the US outcome [i.e., periventricular leukomalacia (PVL), intra-ventricular haemorrhage (IVH) > II grade, hydrocephalus,] and/or congenital malformations; and (c) no indication of visual [retinopathy of prematurity (ROP) > II grade, blindness] or hearing impairment [mono or bilateral hearing loss evaluated by otoacoustic emissions]. Some infants included in the sample had medical complications (see Table 1 for the biological, socio-demographic and medical characteristics of the ELGA sample).

The comparison group included 20 healthy FT infants, who had experienced normal birth (GA $>37$ weeks and BW $\geq 2500 \mathrm{~g}$ ), had no perinatal asphyxia, no history of major cerebral damage and/or congenital malformations, or visual or hearing impairments (see Table 1 for the biological, socio-demographic and medical characteristics of the FT sample). They were recruited from two hospitals in two regions in the center of Italy: Emilia-Romagna (at the same hospital where the preterm newborns were recruited) and Abruzzo (at Chieti Hospital). Chi-square, Fisher exact test and independent sample $t$-tests revealed no differences between ELGA and FT infants on gender, birth order, mother's age and level of education, or presence of twins. 


\subsection{Procedure}

Infants were evaluated at 6,9 and 24 months using a longitudinal design. As in many studies of preterm infants' development in the first 2 years of life, ELGA infants' age was corrected in order to take into account their level of neuropsychological maturation (Johnson \& Marlow, 2006; Sansavini, Guarini, \& Caselli, 2011). The ages of assessment of ELGA infants (6 months corrected age: $M=6.0, S D=0.2 ; 9$ months corrected age: $M=9.1, S D=0.2 ; 24$ months corrected age: $M=24.2, S D=0.4)$ and of the FT sample (6 months: $M=6.2, S D=0.4 ; 9$ months $M=9.1 ; S D=0.3 ; 24$ months: $M=24.5, S D=0.4$ ) were comparable and did not differ significantly.

During the first meeting with the parents, the purpose of the study was explained. At 6 and 9 months, all infants were videotaped for 5 consecutive minutes in interaction with their mothers in a quiet room at the Day-Hospital of the NICU of Bologna University (all ELGA infants and 14 FT infants) or at the Infant Lab of Chieti University (6 FT infants). The mothers were asked to play with their infants as they usually did at home using a set of age-appropriate infant toys that we provided (at 6 months of age: two rattles, three toys for teething, a musical toy, a colourful fruit ring toy; at 9 months of age, in addition to the toys employed at 6 months, some ageappropriate toys were added: a mobile phone, a car, a colourful stacking toy, a spinning top, little animal toys on a revolving base, the farmer says, a doll, a small ball). At 6 months, the mother sat on a chair in front of her infant, who was seated in a highchair at a distance of about $30 \mathrm{~cm}$. At 9 months, the mother could choose the same setting used at 6 months or to sit with her infant on a mat, based on what she typically did at home. At 9 months, 25 mothers preferred to sit on a mat in front of the baby (at a distance of about $30 \mathrm{~cm}$ ); the others sat on a chair facing their infants. Independent sample $t$-tests were conducted in order to test a possible effect of the setting (mat vs. sit on a chair) at 9 months on the main variables considered in the present study. The analyses revealed no significant differences as a function of the position of the dyad (for all, $p>0.05$ ). To make the play interaction as natural as possible, we left the mothers free to select which and how many objects to present to the infant.

The revised Griffiths Mental Developmental Scales 0-2 (GMDS-R, Griffiths, 1996) were administered at 6, 9, and 24 months to all infants by a professional trained psychologist in the presence of the infants' parents. Following instructions of the GMDS-R manual, the psychologist administered each item directly to the infant in order to observe his/her performance and, consequently, assigned it a value. Occasionally, details on some items of the Personal-Social and Hearing \& Language subscales, might have been asked to parents. All test administrations were videotaped, allowing the psychologist to check items completed by infants. Four FT infants were not evaluated at 24 months (two because of infants' illness and two because of change of residence of the family).

This study met ethical guidelines for human subjects protections, including adherence to the legal requirements of the study country, and received formal approval by the local Research Ethical Committee. Moreover, all parents of the ELGA and FT infants gave informed written consent for participation in the study, data analysis, and data publication.

\subsection{Materials}

The revised Griffiths Mental Development Scale 0-2 years (GMDS-R, Griffiths, 1996) was designed for use with children 0-2 years. It requires approximately $45 \mathrm{~min}$ to administer and evaluates five domains: Locomotor sub-scale (LOC, 54 items: gross motor skills including the ability to balance and to control and coordinate movements); Personal-Social sub-scale (PS, 58 items: proficiency in the activities of daily living, level of independence and, social competences); Hearing \& Language sub-scale (HL, 56 items: auditory discrimination and communicative-linguistic comprehension and production); Eye \& Hand Coordination sub-scale (EH, 54 items: fine motor skills, manual dexterity, and visual motor skills); and Performance sub-scale (PERF, 54 items: cognitive functions for planning and completing intentional actions and representing objects mentally). The GMDS-R provides a General Development Quotient of infants' abilities (GQ, $M=100.5, S D=11.8$ ) and five sub-scale quotients (SQ). The GQ and SQ scores are calculated using the tables of standardized scores for the English population since an Italian standardization of this scale is not available. The GMDS-R is frequently used for clinical and research purposes in Neonatology Units in Europe (Johnson \& Marlow, 2006; Sansavini, Guarini, \& Caselli, 2011).

\subsection{Coding}

All exploratory behaviors performed by infants during the 5-min play interaction session were coded from videotapes frame by frame (videos had 24 frames per second) in a single pass by a trained coder blind to infant group membership using a computer-based video interface system (INTERACT version 9, Mangold International GmbH, 2012) that permitted time-intensive coding of the videotapes.

In the current study, we adapted a coding scheme for object exploration designed by Zuccarini et al. (2016) based on a previous study by Ruff et al. (1984). Object Exploration was defined as any engagement with an object that lasted at least one second. It included four mutually exclusive categories: Visual Object Exploration (VOE) occurred when the infant had no physical contact with the object but was looking at it; Motor Object Exploration (MOE) occurred when the infant explores with the hands or the mouth the object while having continuous physical contact with it; No Exploration (NOE) was assigned to episodes in which the infant had no physical or visual contact with the object or in the absence of any object; No code was assigned to frames that could not be coded due to the infant's fussiness or because the infant's behaviors were not visible (e.g., the mother's body partially blocked the camera's view of the child). Since the present study aimed to investigate developmental change in motor exploratory behaviors from 6 to 9 months and the predictive value of specific motor exploratory behaviors on development, only the MOE category was analyzed.

Types of MOE (each lasting at least $1 \mathrm{~s}$ ), identified by Ruff (1984) and Zuccarini et al. (2016) as commonly employed at 6 and 9 
Table 2

Description of the types of MOE (Motor Object Exploration).

\begin{tabular}{ll}
\hline Types of MOE & Description \\
\hline Holding & $\begin{array}{l}\text { Infant holds or touches an object or carries it or his/her hand is resting and open-palmed on it. } \\
\text { Oral Exploration }\end{array}$ \\
$\begin{array}{l}\text { Infant explores with his/her lips, tongue or mouth an object that s/he has brought at mouth with his/her hand (Mouthing). } \\
\text { Infant transfers an object from one hand to another (Transferring) or turns or rotates an object with wrist or finger rotation } \\
\text { (Turn Rotating) or runs his/her fingertips over the surface of the object (Fingering). }\end{array}$ \\
$\begin{array}{l}\text { Manual Rhythmic Exploration } \\
\text { object ox surface with an empty hand or holding another object (Banging). }\end{array}$ \\
\hline
\end{tabular}

months of age were also described. We classified them as Holding, involving a passive exploration with one or both hands; Oral Exploration, involving active exploration with the mouth; Manual Exploration, involving active exploration with the fingers and hands (e.g., turn rotating, fingering, transferring); and Manual Rhythmic Exploration, involving active exploration with the arms and hands (e.g., banging, shaking; see Table 2 for a detailed description).

In addition, to take into account the potential limiting role of maternal behaviors on infants' exploratory behaviors we coded the maternal interruptions (e.g., tendency to interrupt the infant's object exploration by removing the object from his/her hands and directing his/her exploration to another object). No significant differences between the two groups in the frequency per minute of maternal interruptions were revealed by ANOVAs both at 6 [Mothers of ELGA infants: $M=1.15, S D=0.84$; Mothers of FT infants: $M=1.17, S D=0.77 ; F(1,38)=0.004, p=0.95$, partial $\eta^{2}=0.003$ ], and at 9 months [Mothers of ELGA infants: $M=0.37$, $S D=0.03$; Mothers of FT infants: $M=0.04, S D=0.04 ; F(1,38)=0.13, p=0.72$, partial $\left.\eta^{2}=0.003\right]$.

Intercoder reliability was assessed by having a second trained observer who independently coded a randomly selected $20 \%$ of the videorecordings, in equal numbers from the ELGA and FT groups. We used Cohen's Kappa to calculate reliability on the durations of MOE and types of MOE. We obtained a mean value of 0.88 at 6 months (MOE, $K=0.95$; types of MOE, $K=0.82$ ) and a mean value of 0.85 at 9 months (MOE, $K=0.89$; types of MOE, $K=0.81$ ). Values higher than 0.80 indicate almost perfect agreement (Landis \& Koch, 1977).

\subsection{Statistical analyses}

All statistical analyses were carried out using SPSS 21.0 for Windows. Significance level was set at $5 \%$. Data were checked for violation of assumptions using the Kolmogorov-Smirnov and Levene tests.

The temporal duration of each behavioral category was used as the primary dependent measure in the analyses. To control for small variations in session length across groups [at 6 months of age: ELGA: $M=290.05 \mathrm{~s}, S D=26.45 ; \mathrm{FT}: M=299.50 \mathrm{~s} ; S D=2.24$; at 9 months of age: ELGA: $M=294.75 \mathrm{~s}, S D=13.13$; FT: $M=300 \mathrm{~s} ; S D=0$ ], the duration of each category in each session was transformed into a proportional duration by dividing the total duration of each category by the total session duration.

With regard to Aim 1, repeated measure analyses of variances (ANOVAs) were carried out to assess the effect of group (ELGA vs. FT) and age (6 vs. 9 months) on Motor Object Exploration (MOE) and types of MOE (i.e. Holding, Oral, Manual Exploration and Manual Rhythmic Exploration). Post-hoc comparisons were carried out using Bonferroni's method for multiple comparisons. Analyses of variance (ANOVAs) were also run in order to compare the mean scores of ELGA and FT infants on the GMDS-R scores (GQ and SQ subscales) at 6, 9 and 24 months. The Bonferroni correction was applied.

With regard to Aim 2, multiple linear regression analyses (stepwise method with backward variable selection) were performed in order to analyze to which extent Oral and Manual Exploration at 6 months contributed to predict language and cognitive abilities measured with the GMDS-R scores at 24 months, above and beyond neonatal status (ELGA vs. FT infants) and 6-month standardized scores. In the first regression analysis, neonatal status and 6-month HL score were entered first (Model 1) and duration of Oral Exploration at 6 months was added subsequently (Model 2) as predictors of the 24-month HL score. In the second regression analysis, neonatal status and 6-month PERF score were entered first (Model 1) and duration of Manual Exploration was added subsequently (Model 2) as predictors of the 24-month PERF score.

\section{Results}

\subsection{Motor Object Exploration}

Descriptive data on Motor Object Exploration and types of MOE in the ELGA and in the FT groups are reported in Table 3.

With regard to Motor Object Exploration, both groups spent a substantial percentage of time exploring objects passively or actively at both 6 and 9 months (55\% and 71.3\% in ELGA infants; 77.2\% and 75.8\% in FT infants). The ANOVA revealed a significant effect of group membership on MOE $\left[F(1,38)=7.76, p=0.01\right.$, partial $\left.\eta^{2}=0.17\right]$, with FT infants being higher than ELGA infants and a significant age $\times$ group interaction $\left[F(1,38)=6.70, p=0.02\right.$, partial $\left.\eta^{2}=0.14\right]$. Post-hoc comparisons revealed that the percentage of time spent in MOE increased from 6 to 9 months only in ELGA infants $(p=0.006)$ (see Table 3 and Fig. 1).

With regard to Holding, descriptive data showed that this was the manual exploratory behavior most frequently exhibited by both groups at both ages (35.5\% and $42.8 \%$ in ELGA infants; $42.3 \%$ and $52.8 \%$ in FT infants). The ANOVA yielded only a significant age effect $\left[F(1,38)=10.91, p=0.002\right.$, partial $\left.\eta^{2}=0.22\right]$, indicating that the percentage of time spent Holding the object increased from 6 to 9 months (see Table 3 and Fig. 2). 
Table 3

The table summarizes mean, SD, range of proportional durations (\%) of MOE (Motor Object Exploration) and types of MOE: Holding, Oral Exploration, Manual Exploration and Manual Rhythmic Exploration of ELGA (extremely-low gestational age) and FT (full-term) infants at 6 and 9 months (corrected age for ELGA infants)

\begin{tabular}{|c|c|c|c|c|c|c|}
\hline & \multicolumn{3}{|c|}{ ELGA $(n=20)$} & \multicolumn{3}{|c|}{ FT $(\mathrm{n}=20)$} \\
\hline & Mean & $(S D)$ & Range & Mean & $(S D)$ & Range \\
\hline \multicolumn{7}{|l|}{6 months } \\
\hline MOE & 55 & (22) & $12.8-97$ & 77.2 & (13.1) & $37.8-97.6$ \\
\hline Holding & 35.5 & (14.4) & $11.4-60.4$ & 42.3 & (14.7) & $16.6-75.4$ \\
\hline Oral Exploration & 8.7 & (11) & $0-37.2$ & 22.1 & (17.3) & $0-81.8$ \\
\hline Manual Exploration & 5.9 & (7.3) & $0-23.9$ & 7 & (7.6) & $0-29.1$ \\
\hline Manual Rhythmic Exploration & 4.6 & (5.8) & $0-18.3$ & 5.4 & (6.8) & $0-18.1$ \\
\hline \multicolumn{7}{|l|}{9 months } \\
\hline MOE & 71.3 & $(20.2)$ & $22.5-95.3$ & 75.8 & (18.8) & $29.5-100.5$ \\
\hline Holding & 42.8 & (18.8) & $1.9-76.1$ & 52.8 & (16.8) & $21.1-85.6$ \\
\hline Oral Exploration & 9.6 & $(14.6)$ & $0-56$ & 7.7 & $(9.8)$ & $0-31.3$ \\
\hline Manual Exploration & 8.9 & (7.6) & $0-25.5$ & 7.7 & (6.1) & $0.8-20.7$ \\
\hline Manual Rhythmic Exploration & 9.3 & (11.2) & $0-11.2$ & 6.5 & (5.9) & $0-20.1$ \\
\hline
\end{tabular}

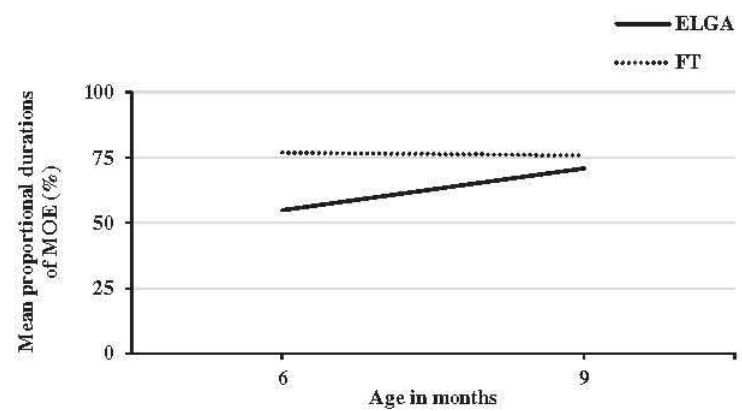

Fig. 1. Longitudinal change of ELGA (extremely-low gestational age) and FT (full-term) infants in MOE (Motor Object Exploration) from 6 to 9 months (corrected age for ELGA infants).

Oral Exploration was present in both samples at 6 and 9 months ( $8.7 \%$ and $9.6 \%$ in ELGA infants; $22.1 \%$ and $7.7 \%$ in FT infants). The ANOVA yielded a significant age effect $\left[F(1,38)=7.76, p=0.008\right.$, partial $\left.\eta^{2}=0.17\right]$ and a significant age $\mathrm{x}$ group interaction $\left[F(1,38)=10.07, p=0.003\right.$, partial $\left.\eta^{2}=0.21\right]$. Post-hoc comparisons revealed that the percentage of time spent mouthing was higher at 6 months and clearly decreased at 9 months in the FT sample $(p=0.003)$, whereas it remained low without significant change in the ELGA sample (see Table 3 and Fig. 2).

Manual Exploration was observed in both samples at 6 and 9 months (5.9\% and 8.9\% in ELGA infants; 7\% and 7.7\% in FT infants) and no group or age differences were found (see Table 3 and Fig. 2). The age $\times$ group interaction was also not significant.

Finally, Manual Rhythmic Exploration was present in both groups of infants at 6 and 9 months ( $4.6 \%$ and $9.3 \%$ in ELGA infants; $5.4 \%$ and $6.5 \%$ in FT infants). The ANOVA showed a significant age effect $\left[F(1,38)=4.1, p=0.046\right.$, partial $\left.\eta^{2}=0.09\right]$, revealing that the time spent in banging and shaking increased from 6 to 9 months (see Table 3 and Fig. 2). All these significant effects were confirmed when excluding the 4 FT infants that could not be evaluated at 24 months.

Finally, we investigated whether the significant pattern of developmental change in object exploration described above held after controlling for gender and maternal educational level, which may identify a further source of variability. Analyses of covariance
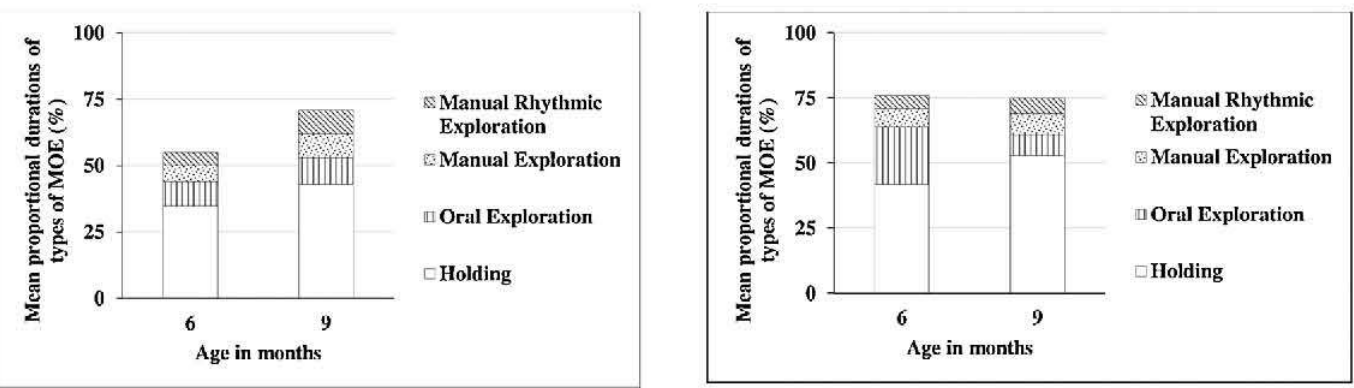

Fig. 2. Mean proportional durations of the types of MOE (Motor Object Exploration) in ELGA (extremely-low gestational age) infants (on the left) and in FT (full-term) infants (on the right) from 6 to 9 months (corrected age for ELGA infants). 
Table 4

The table summarizes $F$, partial eta-squared $\left(\eta^{2}\right)$ values for repeated measure analyses of variance (ANOVAs) and analyses of covariance (ANCOVAs) on proportional durations (\%) of Motor Object Exploration, Holding, Oral Exploration, Manual Exploration, and Manual Rhythmic Exploration. In bold the significant results.

\begin{tabular}{|c|c|c|c|c|c|c|}
\hline & \multicolumn{3}{|c|}{ ANOVA } & \multicolumn{3}{|c|}{ ANCOVA $^{a}$} \\
\hline & $F$ & $p$ & $\pi p^{2}$ & $F$ & $p$ & $\eta p^{2}$ \\
\hline \multicolumn{7}{|c|}{ Motor Object Exploration } \\
\hline Group & 7.76 & 0.010 & 0.166 & 7.56 & 0.009 & 0.174 \\
\hline Age & 3.92 & 0.060 & 0.094 & 3.44 & 0.072 & 0.090 \\
\hline Group $\times$ age & 6.70 & 0.020 & 0.140 & 5.24 & 0.028 & 0.127 \\
\hline \multicolumn{7}{|l|}{ Holding } \\
\hline Group & 3.75 & 0.060 & 0.090 & 3.32 & 0.077 & 0.084 \\
\hline Age & 10.91 & 0.002 & 0.223 & 0.09 & 0.765 & 0.003 \\
\hline Group $\times$ age & 0.35 & 0.560 & 0.009 & 0.23 & 0.631 & 0.006 \\
\hline \multicolumn{7}{|c|}{ Oral Exploration } \\
\hline Group & 2.69 & 0.109 & 0.066 & 2.92 & 0.096 & 0.075 \\
\hline Age & 7.76 & 0.008 & 0.170 & 0.34 & 0.565 & 0.009 \\
\hline Group $\times$ age & 10.07 & 0.003 & 0.210 & 9.63 & 0.004 & 0.211 \\
\hline \multicolumn{7}{|c|}{ Manual Exploration } \\
\hline Group & 0.00 & 0.987 & 0.000 & 0.01 & 0.939 & 0.000 \\
\hline Age & 1.29 & 0.262 & 0.033 & 0.58 & 0.451 & 0.016 \\
\hline Group $\times$ age & 0.48 & 0.494 & 0.012 & 0.40 & 0.532 & 0.011 \\
\hline \multicolumn{7}{|c|}{ Manual Rhythmic Exploration } \\
\hline Group & 0.27 & 0.609 & 0.007 & 0.09 & 0.762 & 0.003 \\
\hline Age & 4.10 & 0.046 & 0.097 & 2.04 & 0.16 & 0.054 \\
\hline Group $\times$ age & 1.53 & 0.224 & 0.039 & 1.12 & 0.296 & 0.030 \\
\hline
\end{tabular}

a ANCOVAs controlling for gender and maternal educational level.

(ANCOVAs) confirmed the significant effects of group membership $\left[F(1,38)=7.56, p=0.009\right.$, partial $\left.\eta^{2}=0.174\right]$, and an age $\mathrm{x}$ group interaction $\left[F(1,38)=5.24, p=0.028\right.$, partial $\left.\eta^{2}=0.127\right]$ on Motor Object Exploration, and an age $\times$ group interaction $[F$ $(1,38)=9.63, p=0.004$, partial $\left.\eta^{2}=0.211\right]$ on Oral Exploration. Conversely, with regard to Holding, Oral Exploration, and Manual Rhythmic Exploration, the age effects observed previously were no longer significant (see Table 4).

\subsection{GMDS-R scores}

Descriptive data on the GMDS-R standard scores at 6, 9 and, 24 months of age in the ELGA and FT groups are presented in Table 5.

ANOVAs revealed significant differences at 6 months between ELGA and FT infants: ELGA infants obtaining lower scores than FT infants on the General Quotient (GQ) as well as on the Performance (PERF) subscales. Differences were also found at 24 months, with ELGA infants lagging significantly behind the FT infants on the General Quotient (GQ) and on the Locomotor (LOC), Eye-Hand Coordination (EH) and Performance (PERF) subscales (see Table 5). The above results obtained at 6 and 9 months were confirmed when excluding the 4 FT infants that could not be evaluated at 24 months.

\subsection{Relationships between exploratory behaviors at 6 months and language and cognitive development at 24 months}

Results of the first multiple linear regression analysis showed that 6-month HL score $(\beta=0.37 . p=0.017)$, but not neonatal status, predicted the 24-month HL score (Model 1) with a $R^{2}$ of $0.16[F(1,34)=7.5, p=0.01]$. When 6-month duration of Oral Exploration $\left(\beta=0.36, p=0.021\right.$ ) was added (Model 2), $R^{2}$ increased to $0.26[F(1,34)=7.2, p=0.002]$. This suggests that duration of Oral Exploration contributed to predict the 24-month HL score, even when accounting for 6-month HL score, and explained a significant additional amount of variation in the dependent variable $(p=0.021)$ (see Table 6).

Similarly, results of the second multiple linear regression analysis showed that 6 -month PERF score $(\beta=0.47, p=0.003)$, but not neonatal status, predicted the 24-month PERF score (Model 1) with a $R^{2}$ of $0.17[F(1,34)=8.3, p=0.007]$. When 6-month duration of Manual Exploration ( $\beta=0.35, p=0.021$ ) was added (Model 2$), R^{2}$ increased to $0.28[F(1,34)=7.7, p=0.002]$. This suggests that duration of Manual Exploration contributed to predict the 24-month PERF score, even when accounting for 6-month PERF score, and explained a significant additional amount of variation in the dependent variable $(\mathrm{p}=0.021$ ) (see Table 6).

\section{Discussion}

The current study yielded two novel contributions. Using a detailed and original micro-analytic coding scheme, different patterns of developmental change in object exploration from 6 to 9 months were revealed in healthy ELGA infants compared to FT infants. In addition, we demonstrated the existence of longitudinal relationships between specific exploratory behaviors at 6 months and cognitive and language development at 24 months. 
Table 5

The table summarizes mean, $\mathrm{SD}$, range, $F, p$ and partial eta-squared $\left(\eta^{2}\right.$ ) values for ANOVAs on General Quotient (GO), Locomotor (LOC), Personal \& Social skills (PS), Hearing \& Language $(\mathrm{HL})$, Eye \& Hand Coordination $(\mathrm{EH})$ and Performance (PERF) standardized scores (GMDS-R) of ELGA (extremely-low gestational age) and FT (full-term) infants at 6,9 and 24 months (corrected age for ELGA infants). In bold the significant results corrected for Bonferroni ( $\mathrm{p} \leq 0.008$ ).

\begin{tabular}{|c|c|c|c|c|c|c|c|c|c|}
\hline & \multicolumn{3}{|c|}{ ELGA $(\mathrm{n}=20)$} & \multicolumn{3}{|c|}{ FT $(\mathrm{n}=20)^{\mathrm{a}}$} & \multicolumn{3}{|c|}{ ANOVA } \\
\hline & Mean & $(S D)$ & Range & Mean & $(S D)$ & Range & $F$ & $p$ & $\pi p^{2}$ \\
\hline \multicolumn{10}{|c|}{6 months } \\
\hline GQ & 103 & (12.1) & $80-130$ & 112.6 & (9.5) & $91-133$ & 7.76 & 0.008 & 0.17 \\
\hline LOC & 98.6 & (17.6) & $57-150$ & 107.9 & (16.9) & $77-142$ & 2.87 & 0.098 & 0.07 \\
\hline PS & 106.4 & (11.2) & $92-132$ & 112.3 & (15.8) & $88-150$ & 1.89 & 0.177 & 0.05 \\
\hline HL & 118.7 & (10.6) & $102-140$ & 120.5 & (12.4) & $88-140$ & 0.24 & 0.625 & 0.01 \\
\hline $\mathrm{EH}$ & 97.4 & (16.5) & $63-123$ & 109.4 & (11.5) & $88-128$ & 7.03 & 0.012 & 0.16 \\
\hline PERF & 93.1 & (18) & 64-117 & 110.4 & (9.1) & $91-126$ & 14.83 & $<0.001$ & 0.28 \\
\hline \multicolumn{10}{|c|}{9 months } \\
\hline $\mathrm{GQ}$ & 103.7 & (14.6) & $76-135$ & 111.1 & (10.6) & $97-130$ & 3.36 & 0.074 & 0.08 \\
\hline LOC & 94.1 & (18.3) & $68-134$ & 105.1 & (17.1) & $79-146$ & 3.89 & 0.056 & 0.09 \\
\hline PS & 107.1 & (22.2) & $67-150$ & 114 & (15.1) & $92-145$ & 1.33 & 0.256 & 0.03 \\
\hline HL & 109.8 & (13.2) & 91-149 & 113.3 & $(8.7)$ & $99-130$ & 0.95 & 0.336 & 0.02 \\
\hline $\mathrm{EH}$ & 106.5 & (20.9) & $65-139$ & 113.4 & (12.3) & $91-129$ & 1.62 & 0.210 & 0.04 \\
\hline PERF & 100 & (17.1) & $68-133$ & 110.9 & (18.5) & $80-150$ & 3.71 & 0.062 & 0.09 \\
\hline \multicolumn{10}{|c|}{24 months } \\
\hline GQ & 85.7 & (16.6) & 50-106 & 105.1 & (8.4) & 87-116 & 18.08 & $<0.001$ & 0.35 \\
\hline LOC & 80.1 & (23) & 50-135 & 112.3 & (22.5) & $60-135$ & 17.78 & $<0.001$ & 0.34 \\
\hline PS & 89.8 & (21.2) & 50-119 & 104.4 & (9.8) & $86-119$ & 6.44 & 0.016 & 0.16 \\
\hline $\mathrm{HL}$ & 90.4 & (19.6) & 50-113 & 103.8 & (14.6) & 59-113 & 5.23 & 0.028 & 0.13 \\
\hline $\mathrm{EH}$ & 87 & (14.6) & 50-107 & 106.4 & (7.4) & $92-123$ & 23.45 & $<0.001$ & 0.41 \\
\hline PERF & 84.3 & (22.1) & 50-111 & 101.8 & (12.7) & 77-118 & 7.92 & 0.008 & 0.19 \\
\hline
\end{tabular}

${ }^{\text {a }}$ Four FT infants were not evaluated at 24 months.

Table 6

Summary of the stepwise regression analyses with backward variable selection for the 24-month $\mathrm{HL}$ score and the 24-month PERF score.

\begin{tabular}{|c|c|c|c|c|c|c|c|c|}
\hline & \multicolumn{3}{|c|}{ Model 1} & \multicolumn{3}{|c|}{ Model 2} & \multicolumn{2}{|c|}{ Variations of $R^{2}$} \\
\hline & B & $\beta$ & $p$ & $B$ & $\beta$ & $p$ & $R^{2}$ & $p$ \\
\hline \multicolumn{9}{|l|}{ 24-month $\mathrm{HL}$ score } \\
\hline 6-month HL score & 0.80 & 0.42 & 0.01 & 0.70 & 0.37 & 0.017 & & \\
\hline Oral Exploration & & & & 0.41 & 0.36 & 0.021 & & \\
\hline \multirow[t]{3}{*}{ Intercept } & -0.64 & & 0.99 & 5.7 & & 0.86 & & \\
\hline & \multicolumn{3}{|c|}{$F_{(1,34)}=7.5 p=0.010 R^{2}=0.16$} & \multicolumn{3}{|c|}{$F_{(1,34)}=7.2 p=0.002 R^{2}=0.26$} & 0.124 & 0.021 \\
\hline & $B$ & $\beta$ & $p$ & $B$ & $\beta$ & $p$ & $R^{2}$ & $p$ \\
\hline \multicolumn{9}{|l|}{ 24-month PERF score } \\
\hline 6-month PERF score & 0.52 & 0.44 & 0.007 & 0.55 & 0.47 & 0.003 & & \\
\hline Manual Exploration & & & & 0.99 & 0.35 & 0.021 & & \\
\hline \multirow[t]{2}{*}{ Intercept } & 39.6 & & 0.040 & 29.9 & & 0.101 & & \\
\hline & \multicolumn{3}{|c|}{$F_{(1,34)}=8.3 p=0.007 R^{2}=0.17$} & \multicolumn{3}{|c|}{$F_{(1,34)}=7.7 p=0.002 R^{2}=0.28$} & 0.121 & 0.021 \\
\hline
\end{tabular}

For the 24-month score, neonatal status and 6-month HL score were entered first (Model 1), and duration of Oral Exploration at 6 months was added subsequently (Model 2). For the 24-month PERF score, neonatal status and 6-month PERF score were entered first (Model 1), and duration of Manual Exploration was added subsequently (Model 2). Neonatal status was not reported in the models since it was not significant.

$P$ value for inclusion in the model was set at 0.1 .

\subsection{Development of object exploration in ELGA and FT infants}

The first major finding of the present study was that developmental change in ELGA infants' Motor Object Exploration differed from that of FT infants between 6 and 9 months. ELGA infants spent lower percentages of time in Motor Object Exploration compared to FT infants, but they increased the time spent exploring objects passively or actively from 6 to 9 months, such that they became more similar to the FT group in the overall duration of Motor Object Exploration by 9 months. By contrast, Motor Object Exploration for the FT group was much higher than for the ELGA group at 6 months and remained stable across this developmental period. These results extend the findings of previous studies (Lobo, Kokkoni et al., 2015; Ruff et al., 1984; Zuccarini et al., 2016) suggesting that ELGA infants without cerebral damage exhibit a delay in Motor Object Exploration at 6 months but appear to recover by 9 months.

Despite the significant increase in time spent in Motor Object Exploration showed by the ELGA group from 6 to 9 months, there were similarities but also differences in patterns of developmental change for specific exploratory behaviors with respect to the FT 
group. One similarity between the ELGA and the FT infants concerned passive exploration, i.e. the Holding behavior. Consistent with Lobo, Kokkoni et al. (2015), ELGA infants, like their FT peers, increased time spent in Holding objects from 6 to 9 months. Since holding can be considered an indicator of attention orientation (Jouen \& Molina, 2005) this result suggests that interest in exploring objects increases both in ELGA and FT infants over time.

The most noteworthy difference between the two groups was in developmental change in active exploration. Our findings revealed that time spent in Oral Exploration remained low and stable in the ELGA group, whereas it was high at 6 months and then decreased from 6 to 9 months in the FT group, as previously found in TD infants (Belsky \& Most, 1981; Lobo et al., 2014; Ruff, 1984). This finding is discrepant with other research that did not find differences in mouthing between low and high risk preterm infants compared to FT infants from birth to 24 months (Lobo, Kokkoni et al., 2015), but it is consistent with previous studies indicating that 8-month-old low risk preterm infants mouthed objects for less time than their FT peers (Kopp, 1974), and that low risk late preterm infants mouthed objects less often than FT infants between 5 and 7 months (Soares, Von Hofsten \& Tudella, 2012). The present study expands those previous findings by demonstrating that ELGA infants show a different pattern of developmental change in Oral Exploration relative to FT infants between 6 and 9 months, since they did not demonstrate a higher duration of Oral Exploration at 6 months than later.

Studies of other populations of infants at risk for motor delays (e.g., younger siblings of children with ASD; Leonard, Elsabaggh, \& Hill, 2013) have also reported shorter durations of Oral Exploration at 6 months compared to infants with no family history of ASD, with no differences between groups at 9 months (Koterba et al., 2013). However, infants can obtain critical information about objects' characteristics, as their shape and structure via Oral Exploration (Ruff et al., 1992). Consequently, spending less time performing oral exploratory behaviors as this ability is emerging implies gathering less object information and reducing learning opportunities from the outset, with potential negative consequences for later development.

With respect to Manual Exploration, we did not find significant differences in relation to group, age, or their interaction. This result is consistent with previous studies that found group differences regarding these specific exploratory behaviors only in high risk preterm infants with neurological damage (Ruff et al., 1984; Lobo, Kokkoni et al., 2015). One similarity between ELGA and FT infants was observed in the developmental change of Manual Rhythmic Exploration, which increased in both groups between 6 and 9 months. As suggested by Palmer (1989), this increase may reflect improvements in control of the arms. This result is partially consistent with Lobo's study (2015), which did not find significant differences in banging objects between FT and low and high risk preterm infants from birth to 24 months. However, those authors also reported that FT infants reached the peak of banging, one of the Manual Rhythmic exploratory behaviors we considered, at 6 months, whereas low risk preterm infants reached the peak quite later, at 24 months. Conversely, we found that both ELGA and FT infants increased Manual Rhythmic Exploration from 6 to 9 months.

Interestingly, age $\mathrm{x}$ group interaction effects observed in Motor Object Exploration and Oral Exploration were maintained when controlling for gender and maternal educational. These findings confirm that the different patterns of developmental change in exploration characterize healthy ELGA infants compared to FT infants.

Scores on the general psychomotor development and in the performance subscale assessed with the GMDS-R were significantly lower in the ELGA group with respect to the FT group at 6 months but no longer at 9 months. This finding suggests that the increasing trend observed in Motor Object Exploration in the ELGA group between 6 and 9 months reflects that of overall psychomotor development. However, differences between the two groups emerged later, at 24 months, when the mean scores of the ELGA group on the GMDS-R were significantly lower than those of the FT group in the Eye \& Hand coordination and Locomotor subscales, as well as in the Performance subscale and in the general psychomotor quotient. These results are consistent with previous studies (Sansavini et al., 2014; Sansavini, Savini, Guarini et al., 2011; Sansavini, Guarini, Savini et al., 2011), showing that alterations associated with premature birth start to emerge in the first year of life, especially in the motor domain, and differences between groups persist in the second year of life, when FT infants consolidated their abilities.

\subsection{Relationships between 6-month exploratory abilities and 24-month language and cognitive development in ELGA and FT infants}

The second aim of this study was to analyze whether the duration of specific Motor Object exploratory behaviors exhibited at 6 months and neonatal status were longitudinally related to language and cognitive development at 24 months. Results showed that the duration of Oral and Manual Exploration explained a portion of variance in the Hearing \& Language subscale score and Performance subscale score at 24 months respectively, in addition to that contributed by 6-month language and cognitive GMDS-R scores respectively. By contrast, neonatal status was not a significant predictor of language and cognitive performance skills at 24 months. These findings raise several issues that should be explored in future research.

First, neonatal status does not fully explain individual differences across domains. Yet, as found in other studies, outcomes of preterm children appeared extremely heterogeneous and may be better predicted by considering a complex interaction among biological and environmental factors, rather than only biological factors (Sansavini, Guarini, Caselli, 2011).

Second, while standardized tools, such as the GMDS-R, may be useful for a clinical evaluation of the preterm population (Johnson \& Marlow, 2006), they provide measures that reflect a general index of development. Therefore, they are not sensitive enough to detect which aspects of a specific ability are impaired. To that aim, a fine-grained observation of infant behaviors that are proven to be relevant for later outcomes would be more effective.

Third, the relationships between proportional durations of Oral and Manual Exploration at 6 months and Hearing \& Language and cognitive Performance scores at 24 months respectively highlight the fact that subtle variations in early skills, such as exploratory behaviours, may have cascading effects on the development of later-emerging complex functions, such as language and cognition. Alternatively, since there is evidence that other early skills (e.g., visual information processing) that are altered in infants born 
preterm (Rose et al., 2005) may have an impact on subsequent outcomes (Bornstein, Hahn, Wolke, 2013), the relationships we found between early exploratory behaviours and later development in multiple psychological domains may be due to a broader range of early difficulties underlying the development of ELGA infants, thus suggesting a continuum of vulnerability across domains. Indeed, closer associations among different domains have been found in studies involving the preterm population (Sansavini, Guarini, \& Caselli, 2011; Johnson, Wolke, Hennessy, \& Marlow, 2011) and other populations with atypical development (Karmiloff-Smith, 2009).

Our findings concerning the relationship between Oral Exploration and language subscale at 24 months bring new evidence to the hypothesis of a cascading effect of mouthing on subsequent language development, which has been observed in typical development (Fagan \& Iverson, 2007). Indeed, several studies have shown that overall fine-motor abilities are related to language and to its developmental precursors (e.g., gestures) in populations at risk for motor and language impairments (LeBarton \& Iverson, 2013), children with ASD and other developmental disorders (Hellendorn et al., 2015), and ELGA infants (Benassi et al., 2016). The present study adds to these results by showing the relative contribution of a specific type of fine-motor skill, i.e. Oral Exploration, to language development.

Concerning the developmental relationship between Manual Exploration and later cognitive development, our findings bring new evidence to data from previous studies. Ruff et al. (1984) found that Manual exploratory behaviors (i.e., fingering, turn rotating, transferring) observed at 9 months were related to the Bayley-I Scales Mental Developmental Index (MDI) at 24 months. We coded the same behaviors as in the above study, finding that this relationship may already be detectable by 6 months of age, earlier than previously observed.

In sum, our findings are consistent with the theoretical assumption that the motor domain is closely linked to the cognitive (Smith, 2005) as well as the language domain (Iverson \& Thelen, 1999; Iverson, 2010). Both cognition and language are influenced by experiences that comes from having a body with particular perceptual and motor capabilities. Therefore, everyday perception-action experiences promote development more than maturation alone (Lobo \& Galloway, 2013; Soska et al., 2010). An implication of this view is that delays or subtle difficulties in early-emerging motor skills, such as those performed in object exploration, may constrain learning taking place during everyday activities, with cascading developmental effects and consequences that extend beyond the motor domain (Thelen, 2005).

\subsection{Limitations}

Limitations that may impact the generalizability of our results should be considered. First, we focused on ELGA infants who were neurologically healthy. Although this is a strength of the research, it limited the sample size, which was relatively small. Nonetheless, we found significant differences between ELGA and FT infants in the developmental changes observed in overall Motor Object Exploration and specific Oral Exploration from 6 to 9 months. Replication of the present findings with larger samples including preterm infants with a less severe grade of prematurity is needed in order to generalize these findings to the entire preterm population and to deepen our understanding of the particular difficulties preterm infants experience with object exploration.

Second, in this study we identified relationships between specific exploratory behaviours and cognitive and language skills assessed with a broad clinical assessment tool, the GMDS-R. It would be interesting to administer more specific standardized cognitive and language tools in order to further understand the contribution of early exploratory behaviours to cognitive and language development.

\section{Conclusions and clinical implications}

In sum, our findings highlighted that extremely preterm birth, without cerebral damage, affects the development of Motor Object Exploration. Relative to their FT peers, ELGA infants showed different patterns of developmental change in Motor Object Exploration and Oral Exploration from 6 to 9 months.

Evidence that object exploration is often delayed in at risk populations (de Campos, Cicuto Ferreira Rocha, \& Savelsbergh, 2009) during the first years of life suggests the importance of studying object exploration skills longitudinally in ELGA infants. It also suggests the need for comparisons between ELGA infants not only to other categories of preterm infants (i.e., those with a less severe degree of prematurity or those with neural damages), but also to other at risk populations, in order to trace back phenotypic differences to their origins in infancy (Karmiloff-Smith, 2007).

Moreover, our findings demonstrate that time spent in active Oral and Manual Exploration at 6 months explains a portion of variance in language and cognitive performance at 24 months. This finding has several implications. First, assessment by a clinical standardized tool is useful but not sufficient to predict cognitive and language development in TD infants as well as in infants at risk for multiple delays, such as infants born preterm. Second, since there is evidence that very preterm infants have the ability to memorize objects by touching them from the first days of life (Marcus et al., 2012), these infants should be monitored as soon as possible, in order to identify potential differences in at risk infants with respect to TD infants in the quantity and quality of Motor Object Exploration. It would contribute to control behaviours that may affect developmental outcomes in various domains. Identification of these differences should be included in the clinical assessment and added to standardized instruments of evaluation. Third, infants with delayed object exploratory skills should be included in intervention programs to support and improve their performance (Lobo, Galloway et al., 2015). Indeed, there are evidences that kangaroo care concurred to ameliorate object exploration abilities of preterm infants (Feldman et al., 2002) or the use of sticky mittens during play with object improves the exploratory abilities of TD infants (Needham et al., 2002). Specific early intervention programs, such as the latter one, may also be useful for preterm infants, 
who have been shown to increase their motor skills (e.g. the ability to explore objects with their feet, after customized programs; Heathcock \& Galloway, 2009), and they could reduce the potential cascading effect that early delays in object exploration may have on other skills.

\section{Funding}

Italian Ministry of Education, University and Research. Grant number: year 2008 - prot. 2008J2WEEK. Grant title: PRIN 2008 "Gestures and language in children with atypical and at risk developmental profiles: relationships among competences, mother-child interaction modalities and proposals of intervention". Coordinator: Alessandra Sansavini.

University of Bologna. Grant number: year 2007-STRAT07SAN. Grant title: Strategic Project 2007 "Early communicative-linguistic and cognitive abilities: risks linked to preterm birth". Coordinator: Alessandra Sansavini.

We are grateful to Cucciolo Associazione in Bologna dei Genitori dei Bambini nati pretermine (Association in Bologna of parents of preterm children) for funding the clinical activity and psychological research at the Unit of Neonatology of Bologna University.

\section{Declaration of interest}

The authors report no conflicts of interest.

\section{Acknowledgements}

We are grateful to the parents and their infants for their participation in this study. We are grateful to Dora Scarlatella for her help with data coding.

\section{References}

Anderson, P. J., \& Doyle, L. W. (2008). Cognitive and educational deficits in children bom extremely preterm. Seminars in Perinatology, 51-58.

Baumgartner, H. A., \& Oakes, L. M. (2013). Investigating the relation between infants' manual activity with objects and their perception of dynamic events. Infoncy, 18 , 983-1006.

Belsky, J., \& Most, R. K. (1981). From exploration to play: A cross-sectional study of infant free play behavior. Developmental Psychology, 17, 630-639.

Benassi, E., Savini, S., Iverson, J. M., Guarini, A., Caselli, M. C., ... Sansavini, A. (2016). Early communicative behaviors and their relationship to motor skills in extremely preterm infants. Research in Developmental Disabilities, 48, 132-144.

Berthental, B., \& Von Hofsten, C. (1998). Eye, hand and trunk control: The foundation for manual development. Neuroscience ond Biobehavioral Reviews, 22, 515-520.

Bornstein, M. H., Hahn, C. S., \& Suwalsky, J. T. D. (2013). Physically developed and exploratory young infants contribute to their own long-term academic achievement. Psychological Science, 2A, 1906-1917.

Bornstein, M., Hahn, C. S., \& Wolke, D. (2013). Systems and cascades in cognitive development and academic achievement. Child Development, 84, 154-162.

Bushnell, E. W., \& Boudreau, J. P. (1993). Motor development and the mind: the potential role of motor abilities as a determinant of aspects of perceptual development. Child Development, 64, 1005-1021.

de Campos, A. C., Cicuto Ferreira Rocha, N. A, \& Savelsbergh, G. J. P. (2009). Reaching and grasping movements in infant at risk: A review. Research in Developmental Disabilities, 30, 819-826.

de Kievet, J. K., Piek, J. P., Aarnoudse-Moens, C. S., \& Oosterlaan, J. (2009). Motor development in very preterm and very low-birth-weight children from birth to adolescence: A meta-analysis. Joumal of American Medical Assoctation, 302, 2235-2242.

Fagan, M. K., \& Iverson, J. M. (2007). The influence of mouthing on infant vocalization. Infoncy, 11, 191-202.

Feldman, R., Weller, A., Sirota, L., \& Eidelman, A. I. (2002). Skin-to-skin contact (kangaroo care) promotes self-regulation in premature infants: Sleep-wake cyclicity, arousal modulation, and sustained exploration. Developmental Psychology, 38, 194-207.

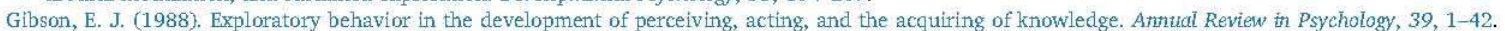

Gomella, T. L. (2009). Neonatology (6th ed.). New York, NY, USA: McGraw Hill Medical.

Griffith, R. (1996). Griffiths mental developmental scale-revised: Birth to 2 years (GMDS-R). Hogrefe.

Heathcock, J. C., \& Galloway, J. C. (2009). Exploning objects with feet advances movement in infants born preterm: A randomized controlled trial. physical Thercoy, 89 , $1027-1038$.

Hellendorn, A., Wijnroks, L, van Daalen, E., Dietz, C., Buitelaar, J. K., \& Leseman, P. (2015). Motor functioning, exploration, visuospatial cognition and language development in preschool children with autism. Research in Developmental Disabilities, 39, 32-42

Hille, E. T. M., Weisglas-Kuperus, N., van Goudoever, J. B., Jacobusse, G. W., ens-Dokkum, M. H., ... Verloove-Vanhoric, S. P. (2008). Functional outcomes and participation in young adulthood for very preterm and very low birth weight infants: the Dutch project on preterm and small for gestational age infants at 19 years of age. Pediatrics, $120,587-595$.

Husby, I. M., Skranes, J., Olsen, A., Brubakk, A. M., \& Evensen, K. A. I. (2013). Motor skills at 23 years of age in young adults born preterm with very low birth weight. Earty Human Development, 89, 747-754.

Iverson, J. M., \& Thelen, E. (1999). Hand, mouth and brain. The dynamic emergence of speech and gesture. Joumal of Conseiousness Studies, 6, 19-40.

Iverson, J. M. (2010). Developing language in developing body: the relationship between motor development and language development. Joumal of Child Language, 37 , 229-261.

Johnson, S., \& Marlow, N. (2006). Developmental screen or developmental testing? Early Human Development, 82, $173-183$.

Johnson, S., Wolke, D., Hennessy, E., \& Marlow, N. (2011). Educational outcomes in extremely preterm children: Neuropsychological correlates and predictors of attainment. Developmental Netropsychology, 36, 74-95.

Jouen, F., \& Molina, M. (2005). Exploration of the newborn's manual activity: A window onto early cognitive processes. Infant Behavior and Development, 28, 227-239. Karmiloff-Smith, A. (2007). Atypical epigenesis. Developmental Sciences, 10, 84-88.

Karmiloff-Smith, A. (2009). Nativism vs: neurocostructivism: rethinking the study of developmental disorders. Developmental Psychology, 45, 56-63.

Kopp, C. (1976). Action schemas of 8-month-old. Developmental Psychology, 12, 361-362

Koterba, E. A., Leezenbaum, N. B., \& Iverson, J. M. (2013). Object exploration at 6 and 9 months in infants with and without risk for autism. Autism, 18, 97-105.

Landis, J. K., \& Koch, G. G. (1977). The measurement of observer agreement for categorical data. Biometrics, 33, $159-174$.

LeBarton, E. S., \& Iverson, J. M. (2013). Fine motor skills predicts expressive language in infant sibilings of children with autism. Develonmental Science, 16, 815-827.

Lederman, S. J., \& Klatzky, R. L. (1993). Extracting object properties through haptic exploration. Acta Psychologica, 84, 29-40.

Leonard, H. C., Elsabbagh, M., \& Hill, E. L. (2013). Early and persistent motor difficulties in infants at-risk of developing autism spectrum disorder: A prospective study. 
European Joumal of Developmental Psychology, 1, 18-35.

Lobo, M. A., \& Galloway, J. C. (2013). The onset of reaching significantly impacts how infant explore both objects and their bodies. Infant Behavior and Development, 36 , $14-24$.

Lobo, M. A., Kokkoni, E., de Campos, A. C., \& Galloway, J. C. (2014). Not just playing around: Infants' behaviors with objects reflects ability, constraints, and object properties. Infant Behavior and Development, 37,334-351.

Lobo, M. A., Galloway, J. C., \& Heathcock, P. T. (2015). Characterization and intervention for upper extremity exploration \& reaching behaviors in infancy. Journal of Hand Therapy, 28, 114-125.

Lobo, M. A., Kolkkoni, E., Cunha, A. B., \& Galloway, J. C. (2015). Infant born preterm demonstrate impaired object exploration behaviors throughout infancy and toddlerhood. Physical Theropy, 95, 51-64.

Marcus, L., Lejeune, F., Beme-Audeoud, F., Gentaz, E., \& Debillon, T. (2012). Tactile sensory capacity of the preterm infant: Manual perception of shape from 28 gestational weeks. Pediatrics, 130, 88-94.

Marlow, N., Wolke, D., Bracewell, M. A., \& Samara, M. (2005). Neurologic and developmental disability at six years of age after extremely preterm birth. The New Engiond Joumal of Medicine, 352, 9-19.

Needham, A, Barrett, T., \& Peterman, K. (2002). A pick-me-up for infants' exploratory skills: Early simulated experiences reaching for objects using sticky mittens enhances young infants' object exploration skills. Infont Behavior and Development, 25, 279-295.

Needham, A. (2000). Improvements in object exploration skills may facilitate the development of object segregation in early infancy. Joumal of Cognition and Development, 1, 131-156.

Oakes, L. M., \& Madole, K. L. (2000). The future of infant categorization research: a process-oriented approach. Child Development, 71, 119-126.

Oudgenoeg-Paz, O., Leseman, P. P. M., \& Volman, C. J. M. (2014). Can infant self-locomotion and spatial exploration predicts spatial memory at school age? European Journal of Developmental Psychology, 11, 36-48.

Palmer, C. F. (1989). The discriminating nature of infants' exploratory actions. Developmental Psychology, 25, 885-893.

Rochat, P., \& Goubet, N. (1995). Development of sitting and reaching in five-to-six months old infants. Infont Behovior and Development, $18,53-68$.

Rochat, P. (1989). Object manipulation and exploration in 2- to 5-month-old infants. Developmental Psychology, 25, 871-884.

Rose, S. A., Feldman, J. F., \& Jankowski, J. J. (2005). Pathways from prematurity and infant abilities to later cognition. Child Development, 76, $1172-1184$.

Ruddy, M. G., \& Bornstein, M. H. (1982). Cognitive correlates of infant attention and maternal stimulation over the first year of life. Child Development, 53, 183-188. Ruff, H. A., McCarton, C., Kurtzberg, D., \& Vaughan, H. G. (1984). Preterm infants manipulative exploration of objects. Child Development, 55, 1166-1173.

Ruff, H. A., Saltarelli, L., Capozzoli, L. M., \& Dubiner, K. (1992). The differentiation of activity in infants' exploration of objects. Developmental Psychology, 28, 851-861.

Ruff, H. A. (1984). Infants' manipulative exploration of objects: effect of age and object characteristics. Developmental Psychology, 20, 9-20.

Sansavini, A, Pentimonti, J., Justice, L., Guarini, A., Savini, S., ... Faldella, G. (2014). Language: motor and cognitive development of extremely preterm children: Modeling individual growth trajectories over the first three years of life. Journal of Comminication Disorders, 49, 55-68.

Sansavini, A., Guarini, A., Savini, S., Broccoli, S., Justice, L., ... Faldella, G. (2011). Longitudinal trajectories of gestural and linguistic abilities in very preterm infants in the second year of life. Netropsychologia, 49, 3677-3688.

Sansavini, A., Guarini, A., \& Caselli, M. C. (2011). Preterm birth: Neuropsychological profliles and atypical developmental pathways. Developmental Disabilities Research Reviews, 17, 102-113.

Sansavini, A., Savini, S., Guarini, A., Broccoli, S., Alessandroni, R., \& Faldella, G. (2011). The effect of gestational age on developmental outcomes: a longitudinal study in the first two years of life. Child Care Health Development, $37,26-36$.

Siegel, L. S. (1981). Infants tests as predictors of cognitive and language development at two years. Child Development, 52, 545-557.

Smith, L. B. (2005). Cognition as a dynamic system: Principles from embodiment. Developmental Review, 25, 278-298.

Soares, D., Von Hofsten, C., \& Tudella, E. (2012). Development of exploratory behavior in late preterm. Infant Behavior and Development, 35, 912-915.

Soska, K. C., Adolph, K. E., \& Johnson, S. P. (2010). System in development: Motor skills acquisition facilitates three-dimensional object completion. Developmental Psychology, 46, 129-138.

Thelen, E. (2005). Dynamic systems theory and the complexity of change. Psychoonalytic Dialogtes, 15, 255-283.

Wilcox, T., Woods, R., Chapa, C., \& McCurry, S. (2007). Multisensory exploration and object individuation in infancy. Developmental Psychology, 43, 479-495.

Wolke, D., \& Meyer, R. (1999). Cognitive status, language attainment: and pre-reading skills of 6 year old very preterm children and their peers: the Bavarian Longitudinal Study. Developmental Medicine and Child Neurology, 41, 94-109.

Zuccarini, M., Sansavini, A., Iverson, J. M., Savini, S., Guarini, A., ... Aureli, T. (2016). Object engagement and manipulation in extremely preterm and full term infants at 6 months of age. Research in Developmental Disabilities, 55, 173-184. 